\title{
Nonlinear optical techniques for improved data capture in fluorescence microscopy and imaging
}

\author{
David S. Bradshaw, Jamie M. Leeder and David L. Andrews* \\ Nanostructures and Photomolecular Systems, School of Chemistry, \\ University of East Anglia, Norwich NR4 7TJ, United Kingdom
}

\begin{abstract}
Multiphoton fluorescence microscopy is now a well-established technique, currently attracting much interest across all fields of biophysics - especially with regard to enhanced focal resolution. The fundamental mechanism behind the technique, identified and understood through the application of quantum theory, reveals new optical polarization features that can be exploited to increase the information content of images from biological samples. In another development, based on a newly discovered, fundamentally related mechanism, it emerges the passage of off-resonant probe laser pulses may characteristically modify the intensity of single-photon fluorescence, and its associated optical polarization behavior. Here, the probe essentially confers optical nonlinearity on the decay transition, affording a means of optical control over the fluorescent emission. Compared to a catalogue of other laser-based techniques widely used in the life sciences, most suffer limitations reflecting the exploitation of specifically lifetime-associated features; the new optical control mechanism promises to be more generally applicable for the determination of kinetic data. Again, there is a prospect of improving spatial resolution, non-intrusively. It is anticipated that tight directionality can be imposed on single-photon fluorescence emission, expediting the development of new imaging applications. In addition, varying the optical frequency of the probe beam can add another dimension to the experimental parameter space. This affords a means of differentiating between molecular species with strongly overlapping fluorescence spectra, on the basis of their differential nonlinear optical properties. Such techniques significantly extend the scope and the precision of spatial and temporal information accessible from fluorescence studies.
\end{abstract}

Keywords: Fluorescence, optical polarization, two-photon, multiphoton, optical nonlinearity, imaging, microscopy, microdomain

\section{INTRODUCTION}

Fluorescence microscopy is a powerful imaging technique exploiting the inherent property that chromophore-containing materials emit detectable, often visible light in response to the absorption of a wavelength-tuned input. Typically each chromophore or fluorophore responsible for producing a signal experiences both the absorption and subsequent emission of a single photon; the latter process is commonly preceded by a degree of intramolecular vibrational relaxation, and hence the output is distinguishable from the input as having a longer wavelength. Single-photon fluorescence imaging techniques have been widely demonstrated within a biological context, where optical discrimination between the input and output makes it possible to identify cellular components in-vivo, generally to a good degree of positional specificity. As an alternative to confocal microscopy, the recently improved accessibility of tunable, ultrafast laser sources has spurred the development and wide application of more specialized fluorescence microscopy techniques based on multiphoton molecular excitation, where electronic excitation and subsequent fluorescence emission is initiated by the simultaneous absorption of two or more input photons. A significant advantage of adopting a multiphoton imaging technique is that molecular excitation occurs within comparatively small cross-sectional areas, the technique requiring a highly focused excitation probe that affords a significantly improved imaging contrast to sub-cellular resolution, simultaneously removing the need for an optical pinhole. ${ }^{1-3}$

High quality images resolved in three dimensions prove invaluable in investigating cellular or sub-cellular systems, making it possible to discern the location, concentration and structure of specific molecular species by fluorescence-

*david.andrews@physics.org 
based discrimination. Multiphoton techniques in particular also prove capable of evaluating time-resolved dynamics, monitoring variations in the comparative orientations, densities and arrangements of key chromophores that have an important role in determining cell and protein function. ${ }^{4-6}$ Although detailed information on the degree of orientational order in specific cellular domains, or the lifetimes associated with the rotational motions of individual fluorophores, is in principle accessible from polarization-resolved measurements, there are significant aspects which, to our knowledge, have seldom been explored in dynamic biological multiphoton imaging. In the first of the topics to be addressed in this paper, we present a theoretical account of two-photon fluorescence, delivering by robust quantum electrodynamical (QED) derivation the detailed results required for any such investigation. The outcomes address the possibility of establishing, from the expected polarization properties of a fluorescence signal generated by a system of chromophores, the degree of local order, ranging between the extremes of fixed and random orientation in three dimensions. The polarization properties of the generated fluorescence signal can register change in the degree of orientational order within the bulk system as micro-domains within the system fluctuate. The rotationally averaged results determine the fully disordered limit of a dynamic spectrum, providing a means in which two-photon imaging can be further developed as a diagnostic tool to monitor variations in intracellular chromophore orientation, for example in response to biological function or controlled external stimuli.

It is widely perceived that the main issue with multiphoton methods is a signal strength that rapidly diminishes with the number of photons in the initial excitation. While the issue of decreasing signal strength can to some extent be offset by chemical means, for example by developing dyes and fluorescence tags with larger multiphoton absorption crosssectional areas, there is an alternative all-optical technique that could enable the fluorescence signal to be substantially enhanced without chemical intervention. The prospect of such an advantage should be most welcome, owing to the fact that foreign fluorescent material can significantly modify or even compromise the properties of a dynamic biological system. In the second topic of this paper, we furnish details of a new method whereby enhanced capture of the emission signal is achieved by the input of an intense, off-resonant laser beam, which may strongly modify the intensity of fluorescence. Here, there is no net absorption or stimulated emission, only elastic forward-scattering - probe laser photons are annihilated and created into the same radiation mode, which thus emerges unchanged. We also show that, through this same optically nonlinear mechanism, there is a sound prospect of improving spatial resolution by nonintrusive means, i.e. it is possible for a tight directionality to be imposed on single-photon fluorescence emission in the short-range region.

In the following section, rotational averaging methods are applied to two-photon fluorescence - the simpler one-photon case being first addressed to introduce and establish the theoretical procedure. Section 3 presents the mechanism to enhance fluorescence intensity, through the input of an off-resonant laser beam. The subject of Section 4 is to analyse the directionality of the fluorescence emission, under the same laser irradiation, as detected by a near-field probe; diagrams are presented to illustrate the results for various input laser intensities. A discussion of the relative merits of these different approaches is given in the concluding Section 5.

\section{MULTIPHOTON FLUORESCENCE}

To generally approach the issues surrounding chromophore orientation or rotational freedom it is appropriate to begin with a representation of the entire optical process, subsuming both the absorption and the emission of fluorescent radiation. Both the absorption and emission stages occur with an efficiency that is determined by the strength of coupling between ground and excited state electronic levels, as determined by components of the relevant transition dipoles or multiphoton tensors. A detailed theoretical representation is therefore to be built on the basis of salient parameters delivered by quantum theory - specifically the quantum amplitudes for initial excitation and the fluorescent decay. In the language of QED, these quantum amplitudes are commonly termed 'matrix elements' because they are in principle derivable for any specified initial and final state; the modulus square of a matrix element for a particular process is proportional to the measurable rate. Once the radiation is regarded as comprising quanta, its absorption signifies the annihilation of one or more photons in the course of photoexcitation; emissive decay is associated with single-photon creation. These events are mutually independent and occur in a step-wise fashion; for our purposes, rotational relaxation following light absorption is assumed to be minimal. To present results that are amenable to 
experimental measurement, matrix elements of the form $M_{f i}(\xi)$ are defined for electronic transitions between initial, $i$, and final, $f$, states in a fluorophore $\xi$. The output signal, $I_{f l u}^{(n)}(\phi)$, is a function of the (experimentally determined) angle between the polarization vector of the incident and emitted light, denoted by $\phi$, and can be described in terms of matrix elements for both $n^{\text {th }}$-order multiphoton absorption and single-photon emission, namely $M_{\alpha 0}^{(n)}(\xi)$ and $M_{0 \alpha}(\xi)$ respectively - in which the molecular labels 0 and $\alpha$ correspond to molecular ground and excited states, i.e.:

$$
I_{f l u}^{(n)}(\phi)=\sum_{\xi} K^{(n)}\left\langle\left|M_{\alpha 0}^{(n)}(\xi)\right|^{2}\left|M_{0 \alpha}(\xi)\right|^{2}\right\rangle \text {. }
$$

The fluorescence signal in equation (1) is thus portrayed in terms of the individual efficiencies of the absorption and emission processes; the constant of proportionality $K^{(n)}$ is itself dependent on a number of experimental parameters including the $n^{\text {th }}$ power of the laser irradiance and the degree of $n^{\text {th }}$ order coherence. To assess the relationship between $I_{f l u}^{(n)}$ and $\phi$ for a fully disordered system in which molecular chromophores (more specifically, the molecular transition moments associated with multiphoton absorption and single photon emission) are randomly oriented relative to the input beam, the angular brackets in equation (1) show the implementation of a rotational averaging procedure, the methods of which follow. To determine rotationally averaged results for one- and two-photon fluorescence, it is first necessary to define the form of all associated matrix elements through the standard methods of QED. ${ }^{7,8}$ Whilst the full derivation is to be presented in detail in a future publication, for present brevity it is appropriate to focus on the important features of each matrix element, namely the relevant electric polarization and molecular transition moment parameters.

\subsection{One-Photon Fluorescence}

To establish the methodology, the optical process of one-photon fluorescence is initially tackled; this procedure involves representing a process in terms of distinct matter-radiation interactions. First is the optical excitation of a molecular chromophore/fluorophore by single-photon absorption, a process which thereby induces an electronic transition from the molecular ground to an accessible excited state configuration of the system. The second interaction signifies molecular relaxation and photon emission, generally returning the molecule to the ground electronic state (though often with a vibrational energy residue). In terms of equation (1), the matrix elements for both one-photon absorption and emission are required. Both are derived from first-order time-dependent perturbation theory, and for the former:

$$
M_{\alpha 0}^{(1)}(\xi) \sim e_{i}^{(l)}(\boldsymbol{p}) \mu_{i}^{\alpha 0}
$$

Here, $e_{i}^{(l)}(\boldsymbol{p})$ or more succinctly $e_{i}^{(l)}$, is a component of the unit electric polarization vector, with $\boldsymbol{p}$ and $l$ respectively denoting the wave-vector and polarization of the input beam. These parameters are distinct from $\boldsymbol{p}^{\prime}$ and $l^{\prime}$, where the prime is introduced to label properties of the output fluorescence, as in the following matrix element for one-photon emission:

$$
M_{0 \alpha}(\xi) \sim{\overline{e_{i}^{\prime}}}^{\left(l^{\prime}\right)} \mu_{i}^{0 \alpha}
$$

In equations (2) and (3), the molecular transition is expressed in terms of transition dipole moment components $\mu_{i}^{\alpha 0}$ and $\mu_{i}^{0 \alpha}$ respectively, the ideal dipole approximation assuming that all higher-order multipolar contributions are comparatively negligible - as is indeed generally the case. In both equations, repetition of the Cartesian index $i$ signifies an assumed summation, a standard convention of tensor analysis that is carried forward in the equations that follow below.

By substituting equations (2) and (3) into (1), and applying a full rotational-average, it emerges that the fluorescence output for one-photon fluorescence can be expressed as follows, $\phi$ being the angle between the input and output polarizations: ${ }^{9}$ 


$$
I_{f l u}^{(1)}(\phi)=\frac{K^{(1)}}{30}\left[\left(T_{\lambda \lambda} \bar{T}_{\mu \mu}+T_{\lambda \mu} \bar{T}_{\mu \lambda}\right)\left(3 \cos ^{2} \phi-1\right)-2\left(T_{\lambda \mu} \bar{T}_{\lambda \mu}\right)\left(\cos ^{2} \phi-2\right)\right]
$$

where the dipole transition moments orientation with respect to their molecular host are denoted by Greek indices; for convenience, notation is introduced in which the unit electric polarization vectors and the molecular transition moments are incorporated within second rank tensors, where for example $S_{i j}$ and $\bar{S}_{i j}$ are equivalent to $e_{i}^{(l)} \bar{e}_{j}^{\left(l^{\prime}\right)}$ and $\bar{e}_{i}^{(l)} e_{j}^{\left(l^{\prime}\right)}$; likewise the molecular transition moments described by $T_{\lambda \mu}$ and $\bar{T}_{\lambda \mu}$ correspond to $\mu_{\lambda}^{\alpha 0} \mu_{\mu}^{0 \alpha}$ and $\bar{\mu}_{\lambda}^{\alpha 0} \bar{\mu}_{\mu}^{0 \alpha}$. In both cases and in all subsequent applications of the notation, the last index in the electric polarization and molecular transition tensors relate to the process of one-photon emission.

\subsection{Two-Photon Fluorescence}

The process of two-photon fluorescence is the most common, experimentally adopted form of multiphoton fluorescence - characterized by the development of three distinct matter-radiation interactions, specifically the concerted absorption of two photons followed by one-photon emission. Whilst the matrix element for one photon emission takes the same form as equation (3), the initial molecular excitation from the ground state progresses through two concurrent photon absorptions, requiring the application of second-order perturbation theory. The matrix element that describes the excitation accounts for the transition through a virtual intermediate state $r$, leading to a summed contribution of transition dipole moment products, $\mu_{i}^{\alpha r} \mu_{j}^{r 0}$ and $\mu_{j}^{\alpha r} \mu_{i}^{r 0}$ :

$$
M_{\alpha 0}^{(2)}(\xi) \sim e_{i}^{(\lambda)} e_{j}^{(\lambda)} \sum_{r} \frac{1}{\left(E_{0 r}+\hbar c p\right)}\left(\mu_{i}^{\alpha r} \mu_{j}^{r 0}+\mu_{j}^{\alpha r} \mu_{i}^{r 0}\right) .
$$

Thus, the ground to excited state transition can proceed through an infinite number of possible intermediate states, whose denominator-weighted sum generates the full matrix element. In practice, the energy denominator term $E_{0 r}$ (the difference between the initial and intermediate molecular energies) usually determines a nearest-resonance condition such that one intermediate state dominates over all other contributions, for example when $E_{r 0}$ comes close to the photon energy $\hbar c p$. The two dipole product contributions in (5) relate to different time-orderings for the two absorbed photons to engage with each transition moment. On the substitution of equations (3) and (5) into (1), and following a rotational-average, the result for two-photon fluorescence is determined as:

$$
\begin{aligned}
I_{f u \mu}^{(2)}(\phi)= & \frac{K^{(2)}}{105}\left[\left(T_{(\lambda \lambda) \mu} \bar{T}_{(\mu \nu) v}+2 T_{(\lambda \mu) \lambda} \bar{T}_{(\mu \nu) v}+T_{(\lambda \mu) \lambda} \bar{T}_{(v v) \mu}+2 T_{(\lambda \mu) v} \bar{T}_{(\lambda v) \mu}\right)\left(3 \cos ^{2} \phi-1\right)\right. \\
& \left.-\left(T_{(\lambda \lambda) \mu} \bar{T}_{(v v) \mu}+2 T_{(\lambda \mu) v} \bar{T}_{\left.(\lambda \mu)_{v}\right)}\right)\left(2 \cos ^{2} \phi-3\right)\right] .
\end{aligned}
$$

Here, brackets around the subscripts denote a symmetrized sum, e.g. $T_{(\lambda \mu) \lambda}=1 / 2\left(T_{\lambda \mu \lambda}+T_{\mu \lambda \lambda}\right)$. Equation (6) is the key result of section 2; more detail on the above expression, and an analysis of three-photon fluorescence are to be presented in other Proceedings (ref. 9).

Due to the molecular complexity of most fluorophores with biological relevance, it is generally beyond the capability of current molecular modeling software to directly calculate the tensor parameters in equation (6). However, it is possible to deploy the above result to assess the degree of order/disorder in a domain from which the fluorescence signal emerges. From this theoretical viewpoint, the initial results appear very promising. By determining how a multiphoton fluorescence signal within a fully disordered system of molecular chromophores responds to an experimental variable specifically the orientation of a signal polarizer relative to the sample - it should prove possible to distinguish and more importantly quantify any degree of localized orientational correlation within a bulk sample as a deviation from the rotationally-averaged response; this is the subject of ongoing research. 


\section{LASER-MODIFIED FLUORESCENCE}

As described above, fluorescence that occurs by spontaneous emission generally involves a single matter-photon interaction as depicted in Fig. 1(a), and its standard theory is cast in terms of first-order time-dependent perturbation theory. When no other light is present - in particular, once any radiation responsible for the initial electronic excitation has passed out of the system, higher order (odd-rank) correction terms are insignificant and denote only self-energy corrections. However this is no longer the case when the fluorophore, in its electronic excited state, is subjected to a throughput of off-resonant laser light - the wavelength of the latter chosen to preclude stimulated emission or any excitation to higher electronic levels. Using a probe laser tuned to a region where the system is transparent, there can be no net absorption or stimulated emission, but elastic forward-scattering events do occur - photons are annihilated and created into the same radiation mode (which thus emerges unchanged). Such events can engage by nonlinear coupling with the fluorescence emission, and the effect is to modify the transition moment for fluorescence decay. This mechanism, represented in Fig. 1(b), entails three matter-photon interactions, i.e. third-order perturbation theory.

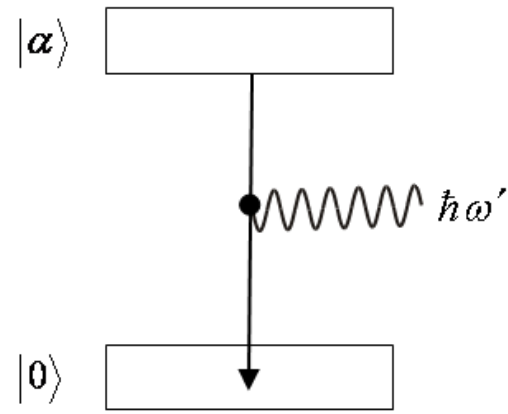

(a)

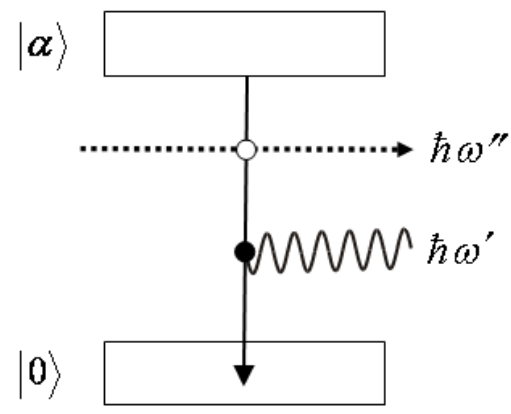

(b)

Fig. 1. (a) Energy level representation for spontaneous fluorescence. Electronic states (and their vibrational manifolds) are signified by the boxes, the wavy line is the emitted fluorescence $\left(\hbar \omega^{\prime}\right)$ and the black vertical arrow is the decay transition: $|0\rangle$ and $|\alpha\rangle$ are the ground and excited states, respectively, the black dot symbolizing a single matter-photon interaction. (b) Same emission but engaging an off-resonant laser beam ( $\left.h \omega^{\prime \prime}\right)$ denoted by the horizontal dashed arrow; the open dot symbolizes two matter-photon interaction (i.e. elastic forward-scattering).

The intensity of fluorescence, $I^{\prime}\left(\Omega^{\prime}\right)$ (power per unit solid angle), which follows from the Fermi Rule rate ${ }^{10}$ multiplied by the energy of a fluorescence photon, $\hbar c k^{\prime} \equiv \hbar \omega^{\prime},{ }^{11}$ is now determined from $I^{\prime}\left(\Omega^{\prime}\right)=2 \pi \rho c k^{\prime}\left|M^{(1)}+M^{(3)}\right|^{2} \square$, where $M^{(1)}$ and $M^{(3)}$ are the quantum amplitudes for the first- and third-order interaction processes, respectively, and the density of radiation states is $\rho=\left(k^{2} V / 8 \pi^{3} \hbar c\right) \mathrm{d} \Omega .^{7}$ The effects to be considered below depend on the relative signs of the first- and third-order amplitudes; a common sign leading to fluorescence enhancement, opposite signs its suppression. To proceed, the following is found for a given emission polarization;

$$
\begin{aligned}
I^{\prime}\left(\Omega^{\prime}\right) & =\left(\frac{c k^{\prime 4}}{8 \pi^{2} \varepsilon_{0}}\right)\left[e_{i}^{\prime} e_{j}^{\prime} \mu_{i}^{0 \alpha} \mu_{j}^{0 \alpha}+\left(I / c \varepsilon_{0}\right) e_{i}^{\prime} e_{j}^{\prime \prime} e_{k}^{\prime \prime} e_{l}^{\prime} \chi_{i j k}^{0 \alpha}\left(\omega^{\prime} ;-\omega^{\prime \prime}, \omega^{\prime \prime}\right) \mu_{l}^{0 \alpha}\right. \\
& \left.+\left(I^{2} / 4 c^{2} \varepsilon_{0}^{2}\right) e_{i}^{\prime} e_{j}^{\prime \prime} e_{k}^{\prime \prime} e_{l}^{\prime} e_{m}^{\prime \prime} e_{n}^{\prime \prime} \chi_{i j k}^{0 \alpha}\left(\omega^{\prime} ;-\omega^{\prime \prime}, \omega^{\prime \prime}\right) \chi_{l m n}^{0 \alpha}\left(\omega^{\prime} ;-\omega^{\prime \prime}, \omega^{\prime \prime}\right)\right],
\end{aligned}
$$

where $\chi_{i j k}^{0 \alpha}$ is a transition hyperpolarizability tensor, $\mathbf{e}^{\prime \prime}$ and $\hbar \omega^{\prime \prime}$ correspond to a probe laser photon, and $I$ is the irradiance of the laser probe. The initial term on the right-hand side in equation (7) corresponds to spontaneous emission - the usual one-photon transition, intrinsic to the system and independent of the probe laser beam - while the last term signifies a coupling of the elastically forward scattered probe beam with the fluorescence emission, a three-photon event. 
The second term, linear in $I$, represents a quantum interference of these two concurrent processes. In general, it may be assumed that the leading term in (7) is non-zero, and the second term a leading correction. The relative sign of this correction will partly depend on the orientations of the relevant transition dipoles relative to the optical polarization vectors; one ensuing consequence of engagement with the probe beam is therefore a modification to the fluorescence anisotropy, an issue discussed below. Typically, assuming the relevant transition dipole components have broadly similar magnitudes and direction, it has previously been determined ${ }^{12}$ that fluorescence can be expected to exhibit a change in intensity of $\sim 10 \%$ for an irradiance of $10^{11} \mathrm{~W} \mathrm{~cm}^{-2}$, and by $\sim 50 \%$ for $I=5 \times 10^{11} \mathrm{~W} \mathrm{~cm}^{-2}$.

Fluorophore photoselection, associated with the use of a plane polarized beam for excitation, is usually the origin of polarized, anisotropic emission. The fluorescence anisotropy, $r$, is determined from the general expression $r=I_{\|}^{\prime}-I_{\perp}^{\prime} / I_{\|}^{\prime}+2 I_{\perp}^{\prime}$, where $I_{\|}^{\prime}$ and $I_{\perp}^{\prime}$ are the intensities measured at $\phi=0$ and $\phi=\pi / 2$, as established by the use of polarizers oriented parallel and perpendicular, respectively, to the polarization vector of the excitation beam. These directions are now chosen as the $z$ - and $x$-directions, respectively. On inclusion of a factor from equation (2) denoting initial one-photon excitation by the polarized beam - and after rotational average, these intensities are expressible as;

$$
\begin{aligned}
& I_{\|}^{\prime}\left(\Omega^{\prime}\right)=\left(\frac{\left|\boldsymbol{\mu}^{0 \alpha}\right|^{2}\left|\boldsymbol{\mu}^{\alpha 0}\right|^{2} c k^{\prime 4}}{40 \pi^{2} \varepsilon_{0}}\right)\left[\frac{2 \cos ^{2} \tau+1}{3}+\frac{C I\left(\cos ^{2} \tau+2\right)}{21 c \varepsilon_{0}}\right], \\
& I_{\perp}^{\prime}\left(\Omega^{\prime}\right)=\left(\frac{\left|\boldsymbol{\mu}^{0 \alpha}\right|^{2}\left|\boldsymbol{\mu}^{\alpha 0}\right|^{2} c k^{\prime 4}}{40 \pi^{2} \varepsilon_{0}}\right)\left[\frac{2-\cos ^{2} \tau}{3}+\frac{C I\left(3-2 \cos ^{2} \tau\right)}{7 c \varepsilon_{0}}\right],
\end{aligned}
$$

where the probe polarization $\mathbf{e}^{\prime \prime}$ is chosen to be oriented in the $x$-direction, and the fluorescence is resolved for polarizations $\mathbf{e}^{\prime}$ in the $z$ - and $x$-directions, respectively. Here, $\tau$ is the angle between the absorption and emission transition moments, the former photoselected by the excitation laser. For the following illustrative purposes, in the construction of equations (8) and (9) the nonlinear transition hyperpolarizabilities are simplified to $C\left|\mu^{0 \alpha}\right|^{3}$ and the final term of equation (1) (quadratically dependent on probe laser intensity) is assumed to be negligible. Deploying the general anisotropy equation, we find:

$$
r=\frac{3 \cos ^{2} \tau-1+\left(C I / c \varepsilon_{0}\right)\left(\cos ^{2} \tau-1\right)}{5+\left(C I / 7 c \varepsilon_{0}\right)\left(20-11 \cos ^{2} \tau\right)} .
$$

In the limiting case $I=0$, the well-known expression ${ }^{13} r=(1 / 5)\left(3 \cos ^{2} \tau-1\right)$ is recovered. Generally, however, a change in fluorescence anisotropy can be anticipated to result from the interaction with the probe beam - although it is to be reemphasized that the state of the latter beam is unaffected. Further analysis of equation (10) shows that, under the specified conditions, the anisotropy $r$ will decrease when the probe produces an increase in fluorescence intensity, whereas when the probe suppresses emission, $r$ increases in value. In the next section, focusing on the same nonlinear mechanism, an analysis of the directionality of single-photon fluorescence is discussed.

\section{DIRECTED FLUORESCENCE}

We now consider, in detail, the spatial characteristics of the fluorescence emerging from a sample whose emission is subjected to the laser modification, as described in the previous Section. For simplicity, we again assume single-photon excitation. It is supposed that the fluorescence is detected by a probe device, sensitive to the emission wavelength, whose variable positioning is employed to analyse the directionality of the emission. By application of an off-resonant laser beam of sufficient intensity, it transpires that the emission field of any excited fluorophore is modified, and the 
transportation of electronic excitation energy from the fluorophore to the probe is augmented. Over typical sample-probe distances this energy transportation takes the form of radiant fluorescence and subsequent absorption; however in the near-field, i.e. over sub-wavelength distances, a modified form of resonance energy transfer (RET) dominates; the latter is our present concern. For arbitrary positions of the probe, relative to the fluorophore source, the power, $P^{\prime}$, acquired by the detector (hence the signal strength) is again determined from the Fermi rule, namely;

$$
P^{\prime}=2 \pi \omega^{\prime} \rho\left|M^{(2)}+M^{(4)}\right|^{2}
$$

where $M^{(2)}$ and $M^{(4)}$ are the quantum amplitudes for second- and fourth-order interaction processes, respectively; both terms are one interaction order larger than those presented earlier, since the theory now accommodates an additional photon detection event at the probe. Specifically, $M^{(2)}$ corresponds to the second-order coupling of conventional (laser throughput-independent) RET, whilst the contribution $M^{(4)}$ represents the optically nonlinear influence of the input beam. In more detail, $M^{(4)}$ involves four mechanistic components: (i) laser photon absorption at source $A$, stimulated emission at probe $B$; (ii) the converse; (iii) absorption and emission at $A$, and; (iv) both photon events at $B$. Accounting for each of these routes for the detector to register a signal, the complete derivation of $M^{(4)}$, as delivered by previous work, ${ }^{14}$ yields the following;

$$
\begin{aligned}
M^{(4)}= & \left(\frac{\left(I_{A} I_{B}\right)^{\frac{1}{2}}}{8 \pi \varepsilon_{0}^{2} c R^{3}}\right) e_{i} e_{l}\left(\delta_{j k}-3 \hat{R}_{j} \hat{R}_{k}\right)\left(\sigma_{i j}^{A}(\omega) \sigma_{l k}^{B}(-\omega)+\sigma_{i j}^{B}(\omega) \sigma_{l k}^{A}(-\omega)\right. \\
& \left.+\chi_{i j l}^{A}(\omega) \mu_{k}^{B}+\chi_{i j l}^{B}(\omega) \mu_{k}^{A}\right),
\end{aligned}
$$

where $R=|\mathbf{R}|$ is the magnitude of the source-probe separation vector, $I_{\xi}$ is the irradiance of the input Gaussian beam at the position of molecule $\xi$, and $\sigma_{i j k}^{\xi}$ and $\chi_{i j k}^{\xi}$ are transition polarizability and hyperpolarizability tensors (denoting twoand three-photon events), respectively. Taking (12), and the commonly known equation of RET (i.e. $M^{(2)}$ ), ${ }^{15}$ an intricate general expression can be determined for the detected power $P^{\prime}$, as is quoted in full elsewhere. ${ }^{16}$ Assuming symmetry selection rules do not exclude any one of the components of $M^{(4)}$, it is still possible by suitable choice of orientation for the off-resonant laser polarization, relative to the molecular system, to achieve suppression of each component. Using this knowledge, two different configurations of particular interest are now examined.

\subsection{Configuration-1}

The first configuration is chosen in such a way that components (i) and (ii) dominate, a condition that can be achieved by setting $\delta=90^{\circ}$ and $\theta=45^{\circ}$, where the angles $\delta$ and $\theta$ denote the orientations of the polarization vectors of the laser beam with respect to $\hat{\mathbf{k}}$ and $\hat{\mathbf{i}}$, respectively. The physical picture is of a throughput beam that propagates in the $z$-direction, perpendicular to the plane in which the probe moves, and centered upon the position of closest approach, i.e. the origin in the $(x, y)$ plane (Fig. 2). With these values employed, the following result is produced:

$$
P^{\prime}=\frac{\omega^{\prime} \mu^{4} \rho}{8 \pi \varepsilon_{0}^{2}\left(r^{2}+z^{2}\right)^{5}}\left\{\frac{\left(I_{A} I_{B}\right)^{\frac{1}{2}} \mu^{2}\left(r^{2}-2 z^{2}\right)}{4 c \varepsilon_{0} \Delta E^{2}}-3 x y\right\}^{2} .
$$

Here, $\mu$ is the magnitude of the transition dipole moment for the source and probe - although, for these calculations, $\mu^{A}$ and $\mu^{B}$ are assumed to be directed in the $\hat{\mathbf{i}}$ and $\hat{\mathbf{j}}$ directions, respectively $-r$ is the magnitude of the projection of particle-probe separation vector, $\mathbf{R}=x \hat{\mathbf{i}}+y \hat{\mathbf{j}}+z \hat{\mathbf{k}}$, on the $x, y$-plane. Moreover, $\Delta E$ is a finite energy which is significantly lower in magnitude than a typical transition energy (explicitly defined elsewhere), ${ }^{16}$ and the contribution from contributions (iii) and (iv) prove to be negligible. The mappings shown in Figs 3(a)-(d) portray $P^{\prime} / \kappa-$ where $\kappa=\omega \mu^{4} \rho / 8 \pi \varepsilon_{0}^{2}-$ as a function of $x$ and $y$ for different values of $I_{0}$; the latter denotes the laser irradiance at the beam 
centre (the associated Gaussian profile being determined by $I_{\xi}=I_{0} \mathrm{e}^{-2(r / w)^{2}}$, where $2 w$ defines the beam waist). The values $\Delta E=5 \times 10^{-20} \mathrm{~J}$ and $w=500 \mathrm{~nm}$ are used in creation of the contour maps. The graphs signify that the shape of the near-field distribution becomes significantly distorted with a sufficient level of input beam irradiance. On introduction of an increasingly powerful off-resonant beam, the four lobes of the conventional cross-polarized fields begin to coalesce and, with increasing $I_{0}$, they eventually merge and compress into a single circular peak centred on the origin. Intermediate stages between the two extremes are shown in Fig. 3 : (a) $I_{0}=3 \times 10^{11} \mathrm{~W}^{\mathrm{cm}^{-2}}$, two diagonally opposite peaks first show convergence towards the origin; (b) $1 \times 10^{12} \mathrm{~W} \mathrm{~cm}^{-2}$, the same peaks begin to merge and the other maxima decrease in magnitude; (c) $2 \times 10^{12} \mathrm{~W} \mathrm{~cm}^{-2}$, the two major peaks fully coalesce and other maxima become diminutive; (d) $3 \times 10^{12} \mathrm{~W} \mathrm{~cm}^{-2}$, the distribution resolves into one elliptical peak at the origin. At higher intensities this peak becomes increasingly circular. The merging of signal maxima is also seen for the case of parallel $\mu^{A}$ and $\mu^{B}$, but here only two peaks are involved.

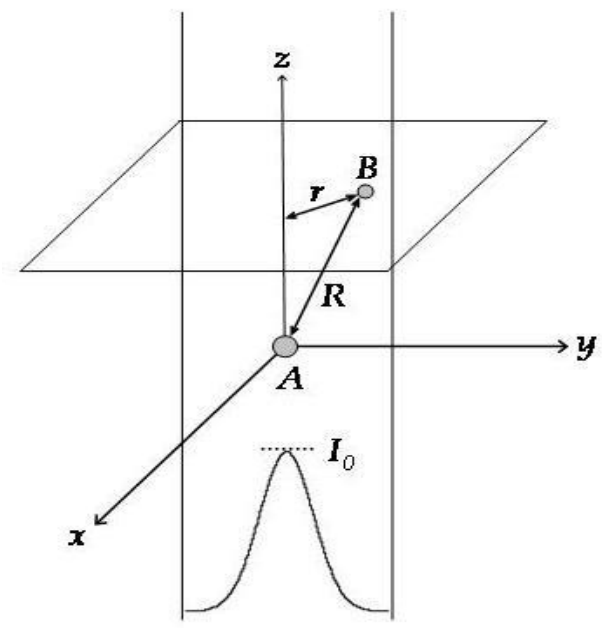

Fig. 2. Geometry of configuration-1 (see text), indicating that the source $A$ and detector $B$ are encompassed within the Gaussian profile of a throughput laser beam propagating along the $z$-axis.

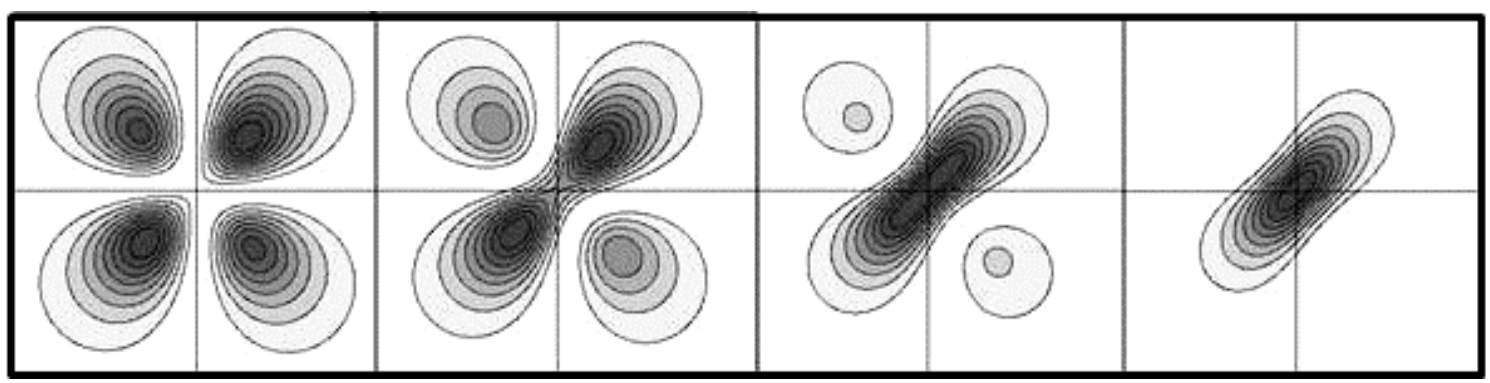

(a)

(b)

(c)

(d)

Fig. 3. Contour maps of $P^{\prime} / K$ against $x$ and $y$ (both in nm) with $z=40 \mathrm{~nm}$, for configuration- 1 (see text) in which $\phi=90^{\circ}$ and $\theta=45^{\circ}$. The input laser irradiance at the beam center, $I_{0}$, is: (a) $3 \times 10^{11} \mathrm{~W} \mathrm{~cm}^{-2}$, (b) $1 \times 10^{12} \mathrm{~W} \mathrm{~cm}^{-2}$, (c) $2 \times 10^{12} \mathrm{~W} \mathrm{~cm}^{-2}$, and (d) $3 \times 10^{12} \mathrm{~W} \mathrm{~cm}$. 


\subsection{Configuration-2}

A second configuration involves setting the angles $\delta=45^{\circ}$ and $\theta=0^{\circ}$, for which contributions (iii) and (iv) are allowed only; the beam propagation is now assumed to be in the $y$-direction, consistent with these values, and for simplicity the centre of the beam is taken as approximately half-way between the source and detector. Using these figures, the following results;

$$
\Gamma^{\prime}=\frac{9 y^{2} \omega^{\prime} \mu^{4} \rho}{8 \pi \varepsilon_{0}^{2}\left(r^{2}+z^{2}\right)^{5}}\left\{x+\frac{\mu^{2} I_{A}(3 z-2 x)}{80 c \varepsilon_{0}(\Delta E)^{2}}\right\}^{2}
$$

With the other parameters given the previously ascribed values, contour maps are again created and shown in Fig. 4: (a)

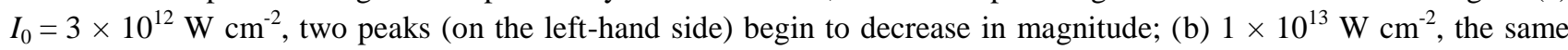
peaks continue to diminish, the other maxima traverse noticeably towards the $y$-axis; (c) $3 \times 10^{13} \mathrm{~W} \mathrm{~cm}^{-2}$, the two remaining peaks continue to move in the direction of negative $x$; (d) $1 \times 10^{14} \mathrm{~W} \mathrm{~cm}^{-2}$, the two maxima become almost symmetric around the $y$-axis. On increasing the irradiance to $1 \times 10^{15} \mathrm{~W} \mathrm{~cm}^{-2}$, the two peaks continue migrating towards lower values of $x$, without losing magnitude; beyond this level of irradiance, no further increase has any discernible effect. From all of these maps it is evident that, with a sufficient level of input beam intensity, nonlinear coupling produces a marked deformation and compression of the near-field. One distinctive aspect of the results for configuration-2 is that a single peak is never seen; the maxima do not coalesce with increasing $I_{0}$. More important, however, is the fact that directional behaviour is once again imposed upon the near-field produced by the source, even though this is to a lesser extent than for configuration-1.

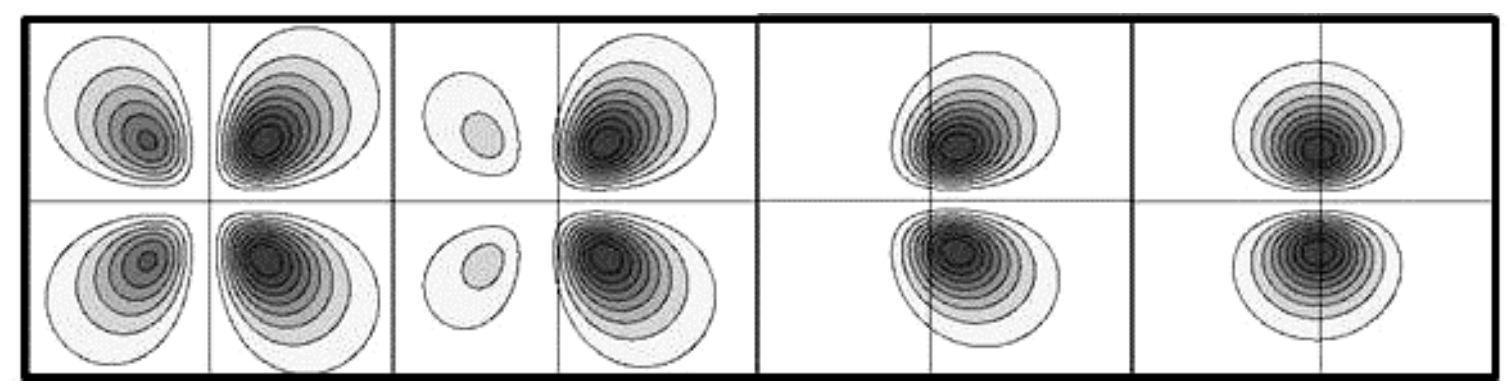

(a)

(b)

(c)

(d)

Fig. 4. As Fig. 3, but for configuration-2 (see text) in which $\phi=45^{\circ}$ and $\theta=0^{\circ}$. The laser irradiance, $I_{0}$, is given by: (a) $3 \times 10^{12}$ $\mathrm{W} \mathrm{cm}{ }^{-2}$, (b) $1 \times 10^{13} \mathrm{~W} \mathrm{~cm}^{-2}$, (c) $3 \times 10^{13} \mathrm{~W} \mathrm{~cm}^{-2}$, and (d) $1 \times 10^{14} \mathrm{~W} \mathrm{~cm}^{-2}$.

\section{DISCUSSION}

The first outcome of the above analysis is the identification, in Section 2, of a dependence of molecular fluorescence on the angular disposition of a polarization plate between the sample and image plate, which should be readily amenable to comparison with multiphoton imaging data. Processing images obtained under different polarization configurations should enable the experimental identification of regions of microscopic order or disorder - this is the subject of further detailed investigations, and another report in the current Proceedings. ${ }^{9}$ The second set of results, reported in Sections 3 and 4, relate to newly discovered effects whereby the throughput of off-resonant light proves to introduce potentially very significant changes to the intensity, polarization and near-field directionality of fluorescent emission. These effects depend on highly specific nonlinear optical properties of the fluorophores, responsible for each signal in a captured fluorescence image. One of the potential advantages of introducing such a phenomenon is to attempt to differentiate between different molecular components, which might normally generate wavelength-similar fluorescent signals. The 
new methodology appears to offer a very promising method of discriminating between such components in an image. Using a tunable laser source, there is also scope to explore the optical dispersion properties of the individual fluorophore nonlinearities. For such a purpose, the titanium:sapphire laser appears to offer an optimal combination of operating characteristics, with high intensities and broad tunability in the near-infrared region. Since the modifications to fluorescence are instantaneous, even the pulsing of such a source might be used with lock-in instrumentation to elicit the modifications in fluorescence behavior.

\section{ACKNOWLEDGEMENTS}

The authors are grateful to both the Leverhulme Trust (DSB) and EPSRC (JML) for financial support, and for funding this research.

\section{REFERENCES}

[1] Rubart, M., “Two-Photon Microscopy of Cells and Tissue,” Circ. Res. 95, 1154-1166 (2004).

[2] Diaspro, A., Bianchini, P., Vicidomini, G., Faretta, M., Ramoino, P. and Usai, C., "Multi-photon excitation microscopy," Biomed. Eng. Online 5, 36-49 (2006).

[3] Oheim, M., Michael, D. J., Geisbauer, M., Madsen, D. and Chow, R. H., "Principles of two-photon excitation fluorescence microscopy and other nonlinear imaging approaches," Adv. Drug Deliver. Rev. 58, 788-808 (2006).

[4] Gratton, E., Barry, N. P., Beretta, S. and Celli, A., "Multiphoton Fluorescence Microscopy," Methods 25, 103-110 (2001).

[5] Lee, P. F., Yeh, A. T. and Bayless, K. J., "Nonlinear optical microscopy reveals invading endothelial cells anisotropically alter three-dimensional collagen matrices," Exp. Cell Res. 315, 396-410 (2009).

[6] Provenzano, P. P., Eliceiri, K. W. and Keely, P. J., "Multiphoton microscopy and fluorescence lifetime imaging microscopy (FLIM) to monitor metastasis and the tumor microenvironment," Clin. Exp. Metastasis 26, 357-370 (2009).

[7] Craig, D. P. and Thirunamachandran, T., [Molecular Quantum Electrodynamics], Dover, New York (1998).

[8] Salam, A., "Molecular quantum electrodynamics in the Heisenberg picture: A field theoretic viewpoint," Int. Rev. Phys. Chem. 27, 405-448 (2008).

[9] Leeder, J. M. and Andrews, D. L., "The analysis of fluorophore orientation by multiphoton fluorescence microscopy," Proc. SPIE 7569 (2010) (in press).

[10] Mandel, L. and Wolf, E., [Optical Coherence and Quantum Optics], University Press, Cambridge, 871 (1995).

[11] Andrews, D. L. and Allcock, P., [Optical Harmonics in Molecular Systems], Wiley-VCH, Weinheim (2002).

[12] Bradshaw, D. S. and Andrews, D. L., "Mechanism for optical enhancement and suppression of fluorescence," J. Phys. Chem. A 113, 6537-6539 (2009).

[13] Lakowicz, J. R., [Principles of Fluorescence Spectroscopy], Kluwer Academic/Plenum, New York, chapter 10 (1999).

[14] Bradshaw, D. S. and Andrews, D. L., "The control of near-field optics: imposing direction through coupling with off-resonant laser light," Appl. Phys. B 93, 13-20 (2008).

[15] Juzeliūnas, G. and Andrews, D. L., [Resonance Energy Transfer], Wiley, Chichester, chapter 2 (1999).

[16] Bradshaw, D. S. and Andrews, D. L., "Optically controlled resonance energy transfer: Mechanism and configuration for all-optical switching,” J. Chem. Phys. 128, 144506 (2008). 